\title{
A New Learning Algorithm for the Hierarchical Structure Learning Automata Operating in the Nonstationary S-Model Random Environment
}

\author{
Norio Baba, Member, IEEE, and Yoshio Mogami, Member, IEEE
}

\begin{abstract}
An extended algorithm of the relative reward strength algorithm is proposed. It is shown that the proposed algorithm ensures the convergence with probability 1 to the optimal path under the certain type of nonstationary environment. Several computer simulation results confirm the effectiveness of the proposed algorithm.
\end{abstract}

Index Terms-Hierarchical structure learning automata (HSLA), nonstationary environment, relative reward strength algorithm.

\section{INTRODUCTION}

$\mathbf{T}$ HE CONCEPT of learning automata (LAs) operating in an unknown environment was initially introduced by Tsetlin [1]. He considered the learning behaviors of finite deterministic automata in a stationary random environment, and succeeded in proving that they are asymptotically optimal under some conditions.

The study of learning behaviors of LAs originated with Varshavskii and Vorontsova [2] and has since been done extensively by many researchers [3]-[26]. LAs have made a significant impact upon many areas of engineering problems; and have so far been successfully applied to many areas. They are expected to provide one of the most powerful tools for constructing an intelligent system.

Although the study of LAs has matured, there are still several problems to be settled. Two of the most important are the low speed of learning, and the insufficient tracking ability to the changing environment (nonstationary environment). In order to overcome the first problem, the concept of the hierarchical structure automata was originally proposed by Thathachar and Ramakrishnan [8] and Ramakrishnan [9]. Since then, the learning behaviors of the hierarchical structure automata have been extensively studied by many researchers [5], [6], [10]-[12]. To overcome the second problem, Thathachar and Sastry [13] proposed a learning algorithm which uses the average of all the reward responses from the environment. They also extended the use of their algorithm to the hierarchical LA model [14],

Manuscript received November 15, 2001; revised March 1, 2002. This work was supported in part by FOST. This paper was recommended by Guest Editors M. S. Obaidat, G. I. Papadimitriou, and A. S. Pomportsis.

N. Baba is with the Department of Information Science, Osaka Kyoiku University, Kashiwara City, Osaka Prefecture 582-8582, Japan.

Y. Mogami is with the Department of Information Science and Intelligent Systems, Faculty of Engineering, University of Tokushima, Tokushima 7708506, Japan.

Publisher Item Identifier S 1083-4419(02)06468-3.
[15]. Following their research, Oommen and Lanctot [16] and Papadimitriou [17] introduced the two concepts of discretized pursuit algorithm and stochastic estimator learning algorithm, respectively. Their continuous work has yielded fruitful results [18], [19].

In 1989, Simha and Kurose [20] derived a very interesting algorithm, the approach of which is considerably different from previous algorithms. They proposed the relative reward strength algorithm, which utilizes the most recent reward response from the environment in an intelligent way. They proved that the proposed algorithm converges to the optimal action with probability 1 . They also gave several computer simulation results which confirmed the effectiveness of their algorithm, and touched upon the possibility of using it efficiently in a certain type of nonstationary environment.

However, despite the effectiveness of their algorithm, additional studies concerning the learning behaviors of this type of algorithm have not followed. We cannot explain such an omission.

One of the most reasonable ways to use this type of algorithm in an environment with high dimensionality is to utilize the hierarchical system of the LAs. This means that one should extend the original relative reward strength algorithm [20] to be utilized in the hierarchical system of the LAs, and then carefully investigate its learning performance. However, unfortunately, this has not yet been attempted.

In this paper, we shall try to extend the algorithm of Simha and Kurose to be used in the hierarchical structure learning automata (HSLA) model. We shall offer evidence that our extended algorithm converges to the optimal action under the certain type of the nonstationary S-model environment. We shall also give several computer simulation results which confirm the effectiveness of our extended algorithm.

\section{BASIC MODEL OF A LEARNING AUTOMATON OPERATING IN AN UNKNOWN ENVIRONMENT}

In the following, we will discuss the learning behaviors of the HSLA operating in the nonstationary S-model environment. To place our study in the appropriate context, we start with a brief explanation of the basic model of the learning mechanism of the single automaton in the stationary random environment.

The learning behaviors of a variable-structure LA operating in an unknown random environment have been discussed extensively under the basic model shown in Fig. 1. Let us 
briefly explain the learning mechanism of the LA "A" under the unknown random environment (teacher environment) $R\left(C_{1}, C_{2}, \ldots, C_{r}\right)$.

The LA "A" is defined by the sextuple $\{S, W, Y, g, P(t), T\} . S$ denotes the set of two inputs $(0,1)$, where " 1 " indicates the reward response from $R\left(C_{1}, C_{2}, \ldots, C_{r}\right)$, and " 0 "indicates the penalty response. (If the set $S$ consists of only the two elements 0 and 1, the environment is said to be a P-model. When the input into A assumes a finite number of values in the closed interval $[0,1]$, it is said to be a Q-model. An S-model is one in which the input into A takes an arbitrary number in the closed line segment $[0,1]$. In this paper, we will deal with the S-model environment.) $W$ denotes the set of $r$ internal states $\left(w_{1}, w_{2}, \ldots, w_{r}\right) ; Y$ denotes the set of $r$ outputs $\left(y_{1}, y_{2}, \ldots, y_{r}\right) ; g$ denotes the output function $y(t)=g[w(t)]$, that is, one-to-one deterministic mapping; $P(t)$ denotes the probability vector $\left[p_{1}(t), p_{2}(t), \ldots, p_{r}(t)\right]^{\prime}$ at time $t$; and its $i$ th component $p_{i}(t)$ indicates the probability with which the $i$ th state $w_{i}$ is chosen at time $t(i=1,2, \ldots, r)$

$$
p_{1}(0)=p_{2}(0)=\cdots=p_{r}(0)=\frac{1}{r}, \quad \sum_{i=1}^{r} p_{i}(t)=1
$$

and $T$ denotes the reinforcement scheme which generates $P(t+$ 1) from $P(t)$.

Suppose that the state $w_{i}$ is chosen at time $t$. Then, the LA "A" performs action $y_{i}$ on the random environment $R\left(C_{1}, C_{2}, \ldots, C_{r}\right)$. In response to the action $y_{i}$, the environment emits output $s(t)=1$ (reward) with probability $1-C_{i}$; and output $s(t)=0$ (penalty) with probability $C_{i}(i=1,2, \ldots, r)$. If all of the $C_{i}(i=1,2, \ldots, r)$ are constant, the random environment $R\left(C_{1}, C_{2}, \ldots, C_{r}\right)$ is said to be a stationary random environment. (The term "single teacher environment" is also used.) On the other hand, if $C_{i}(i=1,2, \ldots, r)$ are not constant, it is said to be a nonstationary random environment. Depending upon the action of the LA "A" and the environmental response to it, the reinforcement scheme $T$ changes the probability vector $P(t)$ to $P(t+1)$.

The values of $C_{i}(i=1,2, \ldots, r)$ are not known in advance. Therefore, it is necessary to reduce the average penalty

$$
M(t)=\sum_{i=1}^{r} p_{i}(t) C_{i}
$$

by selecting an appropriate reinforcement scheme. To judge the effectiveness of an LA operating in a stationary random environment $R\left(C_{1}, C_{2}, \ldots, C_{r}\right)$, various performance measures such as optimality, $\varepsilon$-optimality, absolute expediency, etc., have been set up. (Details are omitted due to space. The reader is referred to [3]-[7].)

In this section, we have briefly introduced the learning mechanism of the single automaton under the stationary random environment $R\left(C_{1}, C_{2}, \ldots, C_{r}\right)$. However, when applying LAs to various actual problems, one often encounters hard situations where nonstationary random environment [5]-[7], [22], [23], multiteacher environment [5], [7], [24], HSLA model [5]-[10],

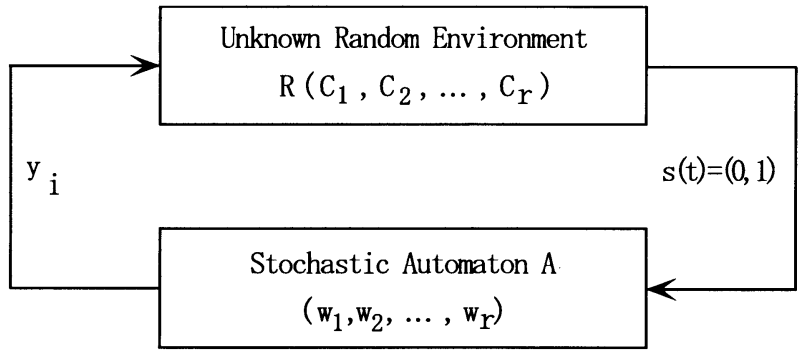

Fig. 1. Basic model of an LA operating in an unknown random environment.

[12], [14], [15], [18], [25], etc., must be considered. In the following section, we will explain the learning mechanism of the HSLA operating in the nonstationary S-model environment.

\section{Hierarchical Structure Learning Automata (HSLA) OPERATING IN THE NONSTATIONARY S-MODEL ENVIRONMENT}

After the pioneering work of Tsetlin [1], learning behaviors of the single automaton have been extensively studied under the basic model shown in Fig. 1. However, one of the most serious bottlenecks concerning the learning model of the single automaton is that its learning performance declines considerably when the space of decisions has high dimensionality.

In order to overcome this problem, Thathachar and Ramakrishnan [8] proposed the concept of the HSLA. Since then, many active researchers have been involved in the study of the learning behaviors of the HSLA.

Next, we consider the learning behaviors of the HSLA operating in the nonstationary S-model environment.

\section{A. Hierarchical Structure Learning Automata (HSLA)}

The system of the HSLA considered in this paper is briefly described as follows. (For easier understanding, the system of the HSLA with the hierarchy of three levels is shown in Fig. 2.) The hierarchy consists of the tree-structured LAs each with $r$ actions. $Z$ denotes the top level (first level) automaton. $Z_{1}, Z_{2}, \ldots, Z_{r}$ denote the automata at the second level. $Z_{11}, Z_{12}, \ldots, Z_{r r-1}, Z_{r r}$ denote the automata at the third level. At the general $s$ th $(s=1,2, \ldots, N)$ level, there are $r^{s-1}$ automata $Z_{i_{1} i_{2} \cdots i_{s-1}}$.

Each LA $Z_{i_{1} i_{2} \cdots i_{s-1}}(s=1,2, \ldots, N)$ is defined by $\left\{\boldsymbol{\alpha}_{i_{1} i_{2} \cdots i_{s-1}}, \beta(t), \quad \boldsymbol{p}_{i_{1} i_{2} \cdots i_{s-1}}(t), T, \boldsymbol{v}_{i_{1} i_{2} \cdots i_{s-1}}(t)\right\}$. Here, $\boldsymbol{\alpha}_{i_{1} i_{2} \cdots i_{s-1}}=\left\{\alpha_{i_{1} i_{2} \cdots i_{s-1} 1}, \alpha_{i_{1} i_{2} \cdots i_{s-1} 2}, \ldots, \alpha_{i_{1} i_{2} \cdots i_{s-1} r}\right\}$ denotes the set of $r$ actions, $\beta(t)$ denotes the reward response from the environment, $\boldsymbol{p}_{i_{1} i_{2} \cdots i_{s-1}}(t)=$ $\left(p_{i_{1} i_{2} \cdots i_{s-1} 1}(t), \quad p_{i_{1} i_{2} \cdots i_{s-1} 2}(t), \ldots, p_{i_{1} i_{2} \cdots i_{s-1} r}(t)\right)^{\prime} \quad$ denotes the action probability distribution, $T$ denotes the reinforcement shceme, $\boldsymbol{v}_{i_{1} i_{2} \cdots i_{s-1}}(t)=\left(v_{i_{1} i_{2} \cdots i_{s-1} 1}(t)\right.$, $\left.v_{i_{1} i_{2} \cdots i_{s-1} 2}(t), \ldots, v_{i_{1} i_{2} \cdots i_{s-1} r}(t)\right)^{\prime}$ denotes the recent reward vector. ${ }^{1}$

Let us now examine how the hierarchical system behaves. Initially, all the action probabilities for each automaton at each level are set equal. The first level automaton $Z$ chooses an action at time $t$ from the action probability distribution $\boldsymbol{p}(t)=$ $\left(p_{1}(t), p_{2}(t), \ldots, p_{r}(t)\right)^{\prime}$. Suppose that $\alpha_{j_{1}}$ is the output from

${ }^{1}$ The recent reward vector will be defined in the next section. 


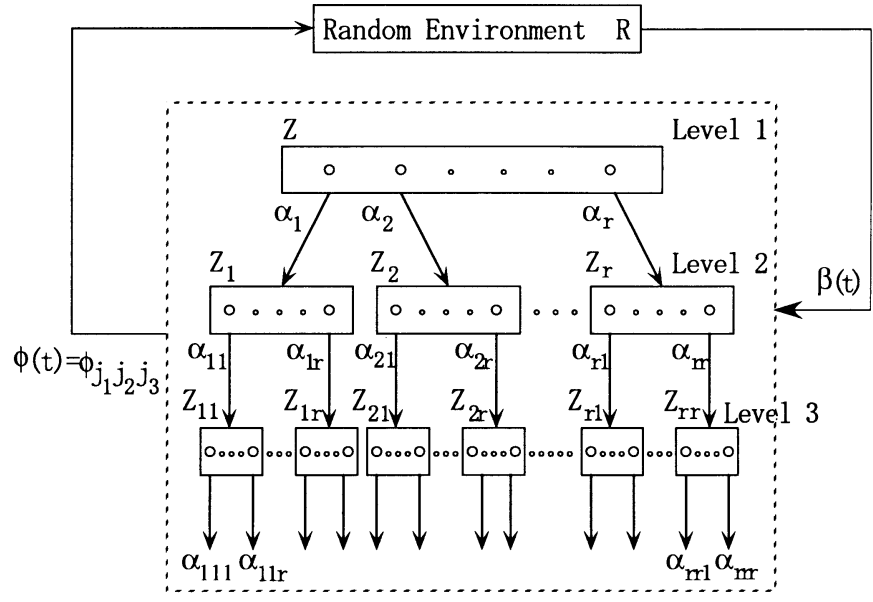

Fig. 2. Hierarchical structure learning automata (HSLA).

$Z$. Then, at the second level, the automaton $Z_{j_{1}}$ is actuated. The $Z_{j_{1}}$ also chooses an action from its action probability distribution. Assume that $\alpha_{j_{1} j_{2}}$ is the output from $Z_{j_{1}}$. Then, at the third level, the automaton $Z_{j_{1} j_{2}}$ is actuated. This cycle of operation is repeated from the top (first level) to the bottom ( $N$ th level). The action at the lowest level interacts with the environment.

The sequence of actions $\left\{\alpha_{j_{1}}, \alpha_{j_{1} j_{2}}, \ldots, \alpha_{j_{1} j_{2} \cdots j_{N}}\right\}$ having been chosen by $N$ automata is called the path. Let $\phi_{j_{1} j_{2} \cdots j_{N}}$ denote the path. Corresponding to the path, the HSLA model receives reward strength $\beta(t)^{2}$ as an environmental response. The HSLA model utilizes this $\beta(t)$ in order to update the current recent reward vector. The action probability vector of each LA relating to the path is updated by using the information concerning the recent reward vector. After all of the above procedures have been completed, time $t$ is set to be $t+1$. By repeating the above cycle of operation, learning by the hierarchical structure automata proceeds. Let $\pi_{j_{1} j_{2} \cdots j_{N}}(t)$ denote the probability that the path $\phi_{j_{1} j_{2} \cdots j_{N}}$ is chosen at time $t$. From this definition

$$
\pi_{j_{1} j_{2} \cdots j_{N}}(t)=p_{j_{1}}(t) p_{j_{1} j_{2}}(t) \cdots p_{j_{1} j_{2} \cdots j_{N}}(t) .
$$

\section{B. The Nonstationary S-Model Environment}

Next, we consider the learning behaviors of the hierarchical structure automata model under the S-model nonstationary environment with the following property.

The optimal path $\phi_{j_{1}^{*} j_{2}^{*} \cdots j_{N}^{*}}$ satisfying the following relation exists uniquely ${ }^{3}$ :

$$
\min _{t} E\left\{\beta_{j_{1}^{*} j_{2}^{*} \cdots j_{N}^{*}}(t)\right\}>\max _{t} E\left\{\beta_{i_{1} i_{2} \cdots i_{N}}(t)\right\}
$$

where $\beta_{i_{1} i_{2} \cdots i_{N}}$ denotes the environmental response corresponding to the path $\phi_{i_{1} i_{2} \cdots i_{N}}$ and $\max \left\{\left|j_{1}^{*}-i_{1}\right|, \mid j_{2}^{*}-\right.$ $\left.i_{2}|, \ldots,| j_{N}^{*}-i_{N} \mid\right\}>0$.

The objective for the hierarchical structure of LA is to find the optimal path $\phi_{j_{1}^{*} j_{2}^{*} \cdots j_{N}^{*}}$ with the probability as high as possible.

$2 \beta(t)$ can be an arbitrary number in the closed line segment $[0,1]$. The large value of $\beta(t)$ indicates that the high reward is given to the sequence of actions being chosen

${ }^{3} E\{\bullet\}$ denotes the mathematical expectation of $\bullet$.
Remark 1: The inequality (2) indicates that the minimum value of the average reward from the environment corresponding to the optimal path is the larger than the maximum value of the average reward corresponding to the other path. This means that we will discuss the learning performance of the proposed algorithm under the nonstationary S-model environment, the constraint of which is rather strict.

\section{A New Learning Algorithm of the HiERARChical STRUCTURE AUTOMATA MODEL}

In 1989, Simha and Kurose [20] proposed a new class of update algorithms which realize a sophisticated use of recent environmental responses. They showed that their algorithms are suitable for tracking nonstationary behaviors of environments. In this section, we shall extend their algorithms in order to use them effectively in the HSLA system.

First, let us define "recent reward to the path" and "recent reward vector."

Definition 1: Let us assume that the path $\phi_{i_{1} i_{2} \cdots i_{N}}$ has been chosen at time $t$ and, corresponding to $\phi_{i_{1} i_{2} \cdots i_{N}}$, reward $\beta_{i_{1} i_{2} \cdots i_{N}}$ has been given from the environment. Then, "recent reward to the path $\phi_{i_{1} i_{2} \cdots i_{N}}$ " is defined as follows:

$$
u_{i_{1} i_{2} \cdots i_{N}}(t)=\beta_{i_{1} i_{2} \cdots i_{N}} .
$$

On the other hand, the other recent reward to the path $\phi_{j_{1} j_{2} \cdots j_{N}}\left({ }^{\exists} k ; i_{k} \neq j_{k}\right)$ is defined as follows:

$$
u_{j_{1} j_{2} \cdots j_{N}}(t)=\beta_{j_{1} j_{2} \cdots j_{N}}\left(\tau_{j_{1} j_{2} \cdots j_{N}}\right)
$$

where $\tau_{j_{1} j_{2} \cdots j_{N}}$ is the most recent time when the path $\phi_{j_{1} j_{2} \cdots j_{N}}$ has been chosen, and $\beta_{j_{1} j_{2} \cdots j_{N}}\left(\tau_{j_{1} j_{2} \cdots j_{N}}\right)$ is the reward from the environment at $\tau_{j_{1} j_{2} \cdots j_{N}}$.

Definition 2: Let $\boldsymbol{v}_{i_{1} i_{2} \cdots i_{s-1}}(t)=\left(v_{i_{1} i_{2} \cdots i_{s-1} 1}(t)\right.$, $\left.v_{i_{1} i_{2} \cdots i_{s-1} 2}(t), \ldots, v_{i_{1} i_{2} \cdots i_{s-1} r}(t)\right)^{\prime}$ be the recent reward vector corresponding to the $s$ th level LA $Z_{i_{1} i_{2} \cdots i_{s-1}}(s=$ $1,2, \ldots, N)$.

Here, each of the components of $\boldsymbol{v}_{i_{1} i_{2} \cdots i_{s-1}}(t)$ is constructed as follows:

at the $N$ th level

$$
v_{i_{1} i_{2} \cdots i_{N}}(t)=u_{i_{1} i_{2} \cdots i_{N}}(t) .
$$

at the $s$ th level $(1 \leq s \leq N-1)$

$$
\begin{array}{r}
v_{i_{1} i_{2} \cdots i_{s}}(t)=\max \left\{v_{i_{1} i_{2} \cdots i_{s} 1}(t), v_{i_{1} i_{2} \cdots i_{s} 2}(t)\right. \\
\left.\ldots, v_{i_{1} i_{2} \cdots i_{s} r}(t)\right\} .
\end{array}
$$

As in [20], we also assume that the following condition holds for all $i_{1}, i_{2}, \ldots, i_{s}\left(i_{q}=1,2, \ldots, r ; q=1,2, \ldots, s(s=\right.$ $1,2, \ldots, N))$ :

$$
q_{\min } \leq p_{i_{1} i_{2} \cdots i_{s}}(t) \leq q_{\max }
$$

where $q_{\min }$ and $q_{\max }$ satisfy the inequalities $0<q_{\min }<$ $q_{\max }<1$ and $q_{\max }=1-(r-1) q_{\min }$, respectively.

Next, we shall propose a new learning algorithm which is an extended form of the relative reward strength algorithm of Simha and Kurose [20] 


\section{A. Learning Algorithm}

Assume that the path $\phi(t)=\phi_{j_{1} j_{2} \cdots j_{N}}$ has been chosen at time $t$ and (corresponding to the output from the hierarchical structure automata system) the environmental response $u_{j_{1} j_{2} \cdots j_{N}}$ has been given. Then, the action probabilities $p_{j_{1} j_{2} \cdots j_{s-1} i_{s}}(t)\left(i_{s}=1,2, \ldots, r\right)$ of each automaton $Z_{j_{1} j_{2} \cdots j_{s-1}}(s=1,2, \ldots, N)$ connected to the path being chosen are updated by the following equation:

$$
\begin{aligned}
p_{j_{1} j_{2} \cdots j_{s-1} i_{s}}(t+1)= & p_{j_{1} j_{2} \cdots j_{s-1} i_{s}}(t) \\
& +\lambda_{j_{1} j_{2} \cdots j_{s-1}}(t) \Delta p_{j_{1} j_{2} \cdots j_{s-1} i_{s}}(t)
\end{aligned}
$$

where $\Delta p_{j_{1} j_{2} \cdots j_{s-1} i_{s}}(t)$ is calculated by

$\Delta p_{j_{1} j_{2} \cdots j_{s-1} i_{s}}(t)$

$$
= \begin{cases}v_{j_{1} j_{2} \cdots j_{s-1} i_{s}}(t) & \\ -\frac{1}{\left|A_{s}(t)\right|} \sum_{l_{s} \in A_{s}(t)} & v_{j_{1} j_{2} \cdots j_{s-1} l_{s}}(t) \\ & { }^{\forall} i_{s} \in A_{s}(t) \\ 0, & i_{s} \notin A_{s}(t) .\end{cases}
$$

Here, the set $A_{s}(t)$ is constructed as follows.

1) Line up $v_{j_{1} j_{2} \cdots j_{s-1} i_{s}}(t)$ in descending order.

2) Set $\quad D_{j_{1} j_{2} \cdots j_{s-1}}=\left\{k_{s} \mid v_{j_{1} j_{2} \cdots j_{s-1} k_{s}}(t)\right.$ $\left.=\max _{i_{s}}\left\{v_{j_{1} j_{2} \cdots j_{s-1} i_{s}}(t)\right\}\right\}$.

3) Repeat the following procedure for $i_{s}\left(i_{s} \notin D_{j_{1} j_{2} \cdots j_{s-1}}\right)$ in descending order of $v_{j_{1} j_{2} \cdots j_{s-1} i_{s}}(t)$ : If the inequality $p_{j_{1} j_{2} \cdots j_{s-1} i_{s}}(t+1)>q_{\min }$ can be satisfied as a result of calculation by (8) and (9), then set $D_{j_{1} j_{2} \cdots j_{s-1}}=$ $D_{j_{1} j_{2} \cdots j_{s-1}} \bigcup\left\{i_{s}\right\}$.

4) Set $A_{s}(t)=D_{j_{1} j_{2} \cdots j_{s-1}}$.

Remark 2: $\lambda_{j_{1} j_{2} \cdots j_{s-1}}(t)$ is the step size parameter at time $t$.

Remark 3: In the proposed algorithm, the change in the action probability of each actuated automaton in the hierarchy is propotional to the difference between the corresponding component of the recent reward vector and the average recent reward over all actions in the actuated automaton.

Remark 4: The action probabilities of each automaton which is not on the selected path are not changed.

Remark 5: As we have already mentioned, the proposed algorithm has been obtained by considering the extension of the relative reward strength algorithm proposed by Simha and Kurose [20]. This means that the proposed algorithm coincides with the original one when $N=1$. Several computer simulation results [20] showed that the relative reward strength algorithm (used in the single automaton) outperforms the $S L_{R-I}$ scheme under the nonstationary random environment with high noise.

\section{CONVERGence THeOrem}

In this section, we shall derive a convergence theorem concerning the learning performance of our proposed algorithm. First, we can obtain the following lemma by paying attention to the components of the reward vector $\mathbf{v}_{j_{1}^{*} j_{2}^{*} \cdots j_{s-1}^{*}}(t)=\left(v_{j_{1}^{*} j_{2}^{*} \cdots j_{s-1}^{*} 1}(t), \quad v_{j_{1}^{*} j_{2}^{*} \cdots j_{s-1}^{*} 2}(t), \ldots\right.$, $\left.v_{j_{1}^{*} j_{2}^{*} \cdots j_{s-1}^{*} r}(t)\right)^{\prime}(s=1,2, \ldots, N)$ corresponding to the optimal path $\phi_{j_{1}^{*} j_{2}^{*} \cdots j_{N}^{*}}$.
Lemma 1: Suppose that each component of the current reward vector is given by (5) and (6). Then, the following inequality concerning the LA $Z_{j_{1}^{*} j_{2}^{*} \cdots j_{s-1}^{*}}(s=1,2, \ldots, N)$ holds:

$$
E\left\{v_{j_{1}^{*} j_{2}^{*} \cdots j_{s}^{*}}(t)\right\}>E\left\{v_{j_{1}^{*} j_{2}^{*} \cdots j_{s-1}^{*} i_{s}}(t)\right\}
$$

where $i_{s}=1,2, \ldots, r, i_{s} \neq j_{s}^{*}$.

Proof: Let us carefully examine each of the LAs relating

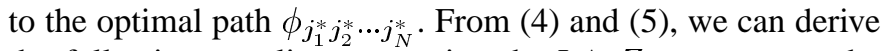
the following equality concerning the LA $Z_{j_{1}^{*} j_{2}^{*} \cdots j_{N-1}^{*}}$ at the lowest level $N$ :

$$
\begin{aligned}
& E\left\{v_{j_{1}^{*} j_{2}^{*} \cdots j_{N-1}^{*} i_{N}}(t)\right\} \\
& =E\left\{u_{j_{1}^{*} j_{2}^{*} \cdots j_{N-1}^{*} i_{N}}(t)\right\} \\
& =E\left\{\beta_{j_{1}^{*} j_{2}^{*} \cdots j_{N-1}^{*} i_{N}}\left(\tau_{j_{1}^{*} j_{2}^{*} \cdots j_{N-1}^{*} i_{N}}\right)\right\} \\
& \quad\left(i_{N}=1,2, \ldots, r\right) .
\end{aligned}
$$

Therefore, from (2) of the optimal path, we can arrive at the following relation:

$$
\begin{aligned}
& E\left\{v_{j_{1}^{*} j_{2}^{*} \cdots j_{N-1}^{*} j_{N}^{*}}(t)\right\} \\
&=E\left\{\beta_{j_{1}^{*} j_{2}^{*} \cdots j_{N-1}^{*}} j_{N}^{*}\left(\tau_{j_{1}^{*} j_{2}^{*} \cdots j_{N-1}^{*} j_{N}^{*}}\right)\right\} \\
&>E\left\{\beta_{j_{1}^{*} j_{2}^{*} \cdots j_{N-1}^{*} i_{N}}\left(\tau_{j_{1}^{*} j_{2}^{*} \cdots j_{N-1}^{*} i_{N}}\right)\right\} \\
&=E\left\{v_{j_{1}^{*} j_{2}^{*} \cdots j_{N-1}^{*} i_{N}}(t)\right\} \\
& \quad\left(i_{N}=1,2, \ldots, r ; i_{N} \neq j_{N}^{*}\right) .
\end{aligned}
$$

Now, let us consider the LA $Z_{j_{1}^{*} j_{2}^{*} \cdots j_{N-2}^{*}}$ at the level $(N-1)$. From (6) and (12), we can get

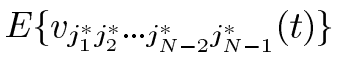

$$
\begin{aligned}
& =\max _{l_{N}}\left\{E\left\{v_{j_{1}^{*} j_{2}^{*} \cdots j_{N-1}^{*} l_{N}}(t)\right\}\right\} \\
& =E\left\{v_{j_{1}^{*}} j_{2}^{*} \cdots j_{N-1}^{*} j_{N}^{*}(t)\right\} \\
& =E\left\{\beta_{j_{1}^{*} j_{2}^{*} \cdots j_{N-1}^{*} j_{N}^{*}}\left(\tau_{j_{1}^{*} j_{2}^{*} \cdots j_{N-1}^{*} j_{N}^{*}}\right)\right\} \\
& E\left\{v_{j_{1}^{*} j_{2}^{*} \cdots j_{N-2}^{*} i_{N-1}}(t)\right\}
\end{aligned}
$$

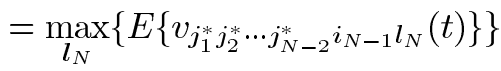

$$
\begin{aligned}
& =E\left\{v_{j_{1}^{*} j_{2}^{*} \cdots j_{N-2}^{*} i_{N-1} k_{N}^{*}}(t)\right\}
\end{aligned}
$$

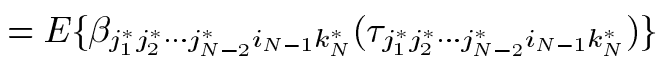

$$
\begin{aligned}
& \left(i_{N-1}=1,2, \ldots, r ; i_{N-1} \neq j_{N-1}^{*}\right) \text {. }
\end{aligned}
$$

If $i_{N-1} \neq j_{N-1}^{*}$, we can show the following relation from (13) and (14):

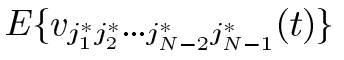

$$
\begin{aligned}
& =E\left\{\beta _ { j _ { 1 } ^ { * } j _ { 2 } ^ { * } \cdots j _ { N - 1 } ^ { * } j _ { N } ^ { * } } \left(\tau_{\left.\left.j_{1}^{*} j_{2}^{*} \cdots j_{N-1}^{*} j_{N}^{*}\right)\right\}}\right.\right.
\end{aligned}
$$

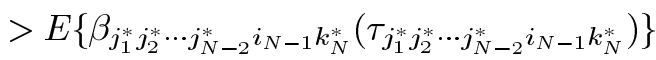

$$
\begin{aligned}
& =E\left\{v_{j_{1}^{*} j_{2}^{*} \cdots j_{N-2}^{*} i_{N-1}}(t)\right\} \\
& \left(i_{N-1}=1,2, \ldots, r ; i_{N-1} \neq j_{N-1}^{*}\right) .
\end{aligned}
$$


Furthermore, let us consider the $\mathrm{LA} Z_{j_{1}^{*} j_{2}^{*} \cdots j_{N-3}^{*}}$ at the level $(N-2)$. From (6), we can get

$$
\begin{aligned}
& E\left\{v_{j_{1}^{*} j_{2}^{*} \cdots j_{N-2}^{*}}(t)\right\} \\
& =\max _{l_{N-1}, l_{N}}\left\{E\left\{v_{j_{1}^{*} j_{2}^{*} \cdots j_{N-2}^{*} l_{N-1} l_{N}}(t)\right\}\right\} \\
& =E\left\{\beta_{j_{1}^{*} j_{2}^{*} \cdots j_{N-2}^{*} j_{N-1}^{*} j_{N}^{*}}\left(\tau_{j_{1}^{*} j_{2}^{*} \cdots j_{N-2}^{*} j_{N-1}^{*} j_{N}^{*}}\right)\right\} \\
& E\left\{v_{j_{1}^{*} j_{2}^{*} \cdots j_{N-3}^{*} i_{N-2}}(t)\right\}
\end{aligned}
$$

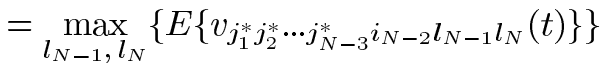

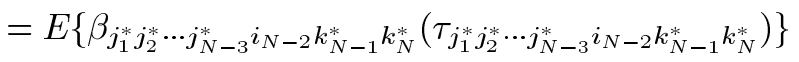

$$
\begin{aligned}
& \left(i_{N-2}=1,2, \ldots, r ; i_{N-2} \neq j_{N-2}^{*}\right) .
\end{aligned}
$$

Therefore, from (2), (16), and (17), we can get the following relation:

$$
\begin{aligned}
& E\left\{v_{j_{1}^{*} j_{2}^{*} \cdots j_{N-2}^{*}}(t)\right\}
\end{aligned}
$$

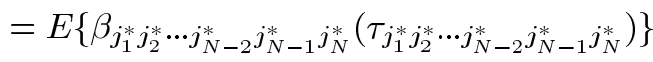

$$
\begin{aligned}
& >E\left\{\beta_{j_{1}^{*} j_{2}^{*} \cdots j_{N-3}^{*} i_{N-2} k_{N-1}^{*} k_{N}^{*}}\left(\tau_{j_{1}^{*} j_{2}^{*} \cdots j_{N-3}^{*} i_{N-2} k_{N-1}^{*} k_{N}^{*}}\right)\right\} \\
& =E\left\{v_{j_{1}^{*} j_{2}^{*} \cdots j_{N-3}^{*} i_{N-2}}(t)\right\} \\
& \left(i_{N-2}=1,2, \ldots, r ; i_{N-2} \neq j_{N-2}^{*}\right) .
\end{aligned}
$$

By repeating the same procedures, we can easily prove that the following inequality holds at an arbitrary level $s(s=1,2, \ldots, N)$

$$
\begin{array}{r}
E\left\{v_{j_{1}^{*} j_{2}^{*} \cdots j_{s}^{*}}(t)\right\}>E\left\{v_{j_{1}^{*} j_{2}^{*} \cdots j_{s-1}^{*} i_{s}}(t)\right\} \\
\left(i_{s}=1,2, \ldots, r ; i_{s} \neq j_{s}^{*}\right) .
\end{array}
$$

Q.E.D.

By taking advantage of Lemma 1, we can obtain the following theorem concerning the convergence to the optimal path $\phi_{j_{1}^{*} j_{2}^{*} \cdots j_{N}^{*}}$.

Theorem 1: Assume that the condition (7) and the conditions given in the lemma hold. Furthermore, let $\lambda_{j_{1} j_{2} \cdots j_{s-1}}(t)$ be a sequence of real numbers such that

$$
\begin{aligned}
\lambda_{j_{1} j_{2} \cdots j_{s-1}}(t)>0, & \sum_{t=1}^{\infty} \lambda_{j_{1} j_{2} \cdots j_{s-1}}(t)=\infty \\
& \sum_{t=1}^{\infty} \lambda_{j_{1} j_{2} \cdots j_{s-1}}^{2}(t)<\infty .
\end{aligned}
$$

Then, the path probability $\pi_{j_{1}^{*} j_{2}^{*} \cdots j_{N}^{*}}(t)$ that the hierarchical structure automata system chooses the optimal path $\phi_{j_{1}^{*} j_{2}^{*} \cdots j_{N}^{*}}$ at time $t$ converges almost surely to $\left(q_{\max }\right)^{N}$.

Proof: From the lemma, it can be shown that $E\left\{v_{j_{1}^{*} j_{2}^{*} \cdots j_{s-1}^{*} j_{s}^{*}}(t)\right\}$ corresponding to the optimal action $\alpha_{j_{1}^{*} j_{2}^{*} \cdots j_{s-1}^{*} j_{s}^{*}}$ (related to the optimal path) is always greater than $E\left\{v_{j_{1}^{*} j_{2}^{*} \cdots j_{s-1}^{*} i_{s}}(t)\right\}$ corresponding to the other action $\alpha_{j_{1}^{*} j_{2}^{*} \ldots j_{s-1}^{*} i_{s}}\left(i_{s} \neq j_{s}^{*}\right)$. This means that the assumption of Theorem 1 [20] concerning the average reward holds. Therefore, it can be shown that by using the same procedure as in Theorem 1 [20]

$$
\lim _{t \rightarrow \infty} p_{j_{1}^{*} j_{2}^{*} \cdots j_{s}^{*}}(t)=q_{\max } \text { with probability } 1
$$

for all of the LAs $Z_{j_{1}^{*} j_{2}^{*} \cdots j_{s-1}^{*}}(s=1,2, \ldots, N)$ related to the optimal path (given in the Appendix). The path probability is thus given by (1). Hence, from (20), we can obtain

$$
\begin{aligned}
& \lim _{t \rightarrow \infty} \pi_{j_{1}^{*} j_{2}^{*} \cdots j_{N}^{*}}(t) \\
& \quad=\lim _{t \rightarrow \infty}\left\{p_{j_{1}^{*}}(t) p_{j_{1}^{*} j_{2}^{*}}(t) \cdots p_{j_{1}^{*} j_{2}^{*} \cdots j_{N}^{*}}(t)\right\} \\
& \quad=\lim _{t \rightarrow \infty} p_{j_{1}^{*}}(t) \lim _{t \rightarrow \infty} p_{j_{1}^{*} j_{2}^{*}}(t) \cdots \lim _{t \rightarrow \infty} p_{j_{1}^{*} j_{2}^{*} \cdots j_{N}^{*}}(t) \\
& \quad=q_{\max } q_{\max } \cdots q_{\max }=\left(q_{\max }\right)^{N} .
\end{aligned}
$$

Q.E.D.

\section{COMPUter Simulation Results}

In order to investigate whether the proposed algorithm can be successfully utilized in the nonstationary S-model environments, we carried out many computer simulations.

We shall show one of the computer simulation results concerning the learning behaviors of the HSLA.

Before going into details concerning the computer simulation, we shall briefly explain the hierarchical automata system, nonstationary environments, etc.

\section{A. Hierarchical Structure Automata Model}

The hierarchical structure automata model is characterized by the following:

1) the number of the levels of the HSLA: 11;

2) the number of the actions of each automaton in the hierarchy: 2;

3) the total number of paths: 2048;

4) the optimal path: $\phi_{11} 111221121$.

\section{B. Nonstationary Environment}

We have considered the following nonstationary environment:

1) The environmental reward $\beta(t)$ at time $t$ corresponding to the output $\phi(t)=\phi_{i_{1} i_{2} \cdots i_{N}}$ from the hierarchy is characterized by the following equation:

$$
\beta(t)=\beta_{i_{1} i_{2} \cdots i_{N}}(t)+e_{i_{1} i_{2} \cdots i_{N}} \xi
$$

where $\xi$ is the random variable with the uniform probability density function in the closed interval $[-0.5,0.5]$ and $\beta_{i_{1} i_{2} \cdots i_{N}}(t)=a_{i_{1} i_{2} \cdots i_{N}}+b_{i_{1} i_{2} \cdots i_{N}} \sin \left(c_{i_{1} i_{2} \cdots i_{N}} \pi t+\right.$ $\left.d_{i_{1} i_{2} \cdots i_{N}}\right)$. Here, $a_{i_{1} i_{2} \cdots i_{N}}, b_{i_{1} i_{2} \cdots i_{N}}, c_{i_{1} i_{2} \cdots i_{N}}, d_{i_{1} i_{2} \cdots i_{N}}$, and $e_{i_{1} i_{2} \cdots i_{N}}$ are the positive scalors, the values of which have been chosen in such a way that the inequality $0 \leq$ $\beta(t) \leq 1$ holds for all $t$. 
TABLE I

VALUES OF THE COEFFICIENTS $a_{i_{1} i_{2} \cdots i_{N}}$ AND $b_{i_{1} i_{2} \cdots i_{N}}$

\begin{tabular}{l|c|c}
\hline path & $\mathrm{a}$ & $\mathrm{b}$ \\
\hline $\begin{array}{l}\text { Optimal Path } \\
\phi_{11111221121}\end{array}$ & 0.88 & 0.05 \\
\hline $\begin{array}{l}\text { Second optimal path } \\
\phi_{1111112122}\end{array}$ & 0.70 & 0.08 \\
\hline
\end{tabular}

TABLE II

SIMULATION RESUlTS (OUR ALGORITHM)

\begin{tabular}{c|c|c}
\hline $\begin{array}{c}\text { Step size } \\
\text { parameters }\end{array}$ & $\begin{array}{c}\text { Average } \\
\text { Number } \\
\text { of Iterations }\end{array}$ & $\begin{array}{c}\text { Percentage } \\
\text { of correct } \\
\text { convergences(\%) }\end{array}$ \\
\hline 0.01 & 2234.0 & 100 \\
0.02 & 1188.2 & 100 \\
0.03 & 851.4 & 100 \\
0.04 & 8640.5 & 100 \\
0.05 & 11944.1 & 100 \\
\hline
\end{tabular}

2) In the simulation, we utilized the following particular combinations concerning the parameter values:

- $a_{i_{1} i_{2} \cdots i_{N}}, b_{i_{1} i_{2} \cdots i_{N}}$ : The values of $a_{i_{1} i_{2} \cdots i_{N}}$ and $b_{i_{1} i_{2} \cdots i_{N}}$ corresponding, respectively, to the optimal path and the second optimal path are given in Table I. The values of the other $a_{i_{1} i_{2} \cdots i_{N}}$ have been chosen by using the random variable with the uniform probability density function in the closed interval $[0.1,0.5]$. On the other hand, the values of the other $b_{i_{1} i_{2} \cdots i_{N}}$ have been chosen to be equal to the same value 0.1 .

- $c_{i_{1} i_{2} \cdots i_{N}}$ : The value of $c_{i_{1} i_{2} \cdots i_{N}}$ has been chosen by using the random variable with the uniform probability density function in the closed interval [0.3, $0.8]$.

- $d_{i_{1} i_{2} \cdots i_{N}}: 0.5$ for all $i_{1}, i_{2}, \ldots, i_{N}$.

- $e_{i_{1} i_{2} \cdots i_{N}}: 0.03$ for all $i_{1}, i_{2}, \ldots, i_{N}$.

\section{Parameters $q_{\min }$ and $q_{\max }$}

As the parameters $q_{\min }$ and $q_{\max }$, we have used the following values:

1) $q_{\min }=0.002$

2) $q_{\max }=0.998$.

We have used the same value of the parameter $\lambda_{i_{1} i_{2} \cdots i_{s-1}}(s=$ $1,2, \cdots, N)$ from the top level of the hierarchy to the bottom level.

Table II shows the average number of iteration and the probability that the proposed algorithm has succeeded in finding the optimal path. In each computer experiment, we have carried out 30 simulations. In order to compare our proposed algorithm with the familiar learning algorithm by Thathachar and Ramakrishnan [8], we have also carried out computer simulations using their algorithm by keeping the same experimental conditions. Table III shows the computer simulation results. From these results, we may confirm the effectiveness of our proposed algorithm.
TABLE III

SiMULATION RESULTS (T\&R ALGORITHM)

\begin{tabular}{c|c|c}
\hline $\begin{array}{c}\text { Step size } \\
\text { parameters }\end{array}$ & $\begin{array}{c}\text { Average } \\
\text { Number } \\
\text { of Iterations }\end{array}$ & $\begin{array}{c}\text { Percentage } \\
\text { of correct } \\
\text { convergences(\%) }\end{array}$ \\
\hline 0.00025 & 127132.4 & 100 \\
0.00026 & 131674.1 & 100 \\
0.00027 & 126888.2 & 91 \\
0.00028 & 126098.2 & 100 \\
0.00029 & 113611.0 & 91 \\
\hline 0.0025 & 11398.4 & 37 \\
0.0026 & 10978.6 & 23 \\
0.0027 & 12277.0 & 30 \\
0.0028 & 11268.2 & 30 \\
0.0029 & 10433.4 & 23 \\
\hline
\end{tabular}

\section{CONCLUSIONS}

In this paper, we have extended the relative reward strength algorithm of Simha and Kurose [20] in order that it can be used in the HSLA model. We have indicated that the proposed algorithm ensures convergence to the optimal action with probability 1 under the certain type of nonstationary S-model environment. Future research is needed to investigate the learning behaviors of the hierarchical structure automata under various types of the nonstationary environments.

\section{APPENDIX}

Here, we give similar definitions to those in [20].

$$
\begin{aligned}
& I_{B}=\left[q_{\min }, q_{\max }-\frac{\varepsilon}{2}\right] \\
& I_{C}=\left[q_{\max }-\frac{\varepsilon}{2}, q_{\max }\right] \\
& L\left(p_{j_{1}^{*} j_{2}^{*} \cdots j_{s}^{*}}(t)\right)=\left\{\begin{aligned}
q_{\max }-\frac{\varepsilon}{2}- & p_{j_{1}^{*} j_{2}^{*} \cdots j_{s}^{*}}(t) \\
& p_{j_{1}^{*} j_{2}^{*} \cdots j_{s}^{*}}(t) \in I_{B} \\
0, & p_{j_{1}^{*} j_{2}^{*} \cdots j_{s}^{*}}(t) \in I_{C} .
\end{aligned}\right. \\
& P_{s B}(t)=\operatorname{Pr}\left(p_{j_{1}^{*} j_{2}^{*} \cdots j_{s}^{*}}(t) \in I_{B}\right) \\
& P_{s C}(t)=\operatorname{Pr}\left(p_{j_{1}^{*} j_{2}^{*} \cdots j_{s}^{*}}(t) \in I_{C}\right) \\
& b_{s}(t)=E\left\{L^{2}\left(p_{j_{1}^{*} j_{2}^{*} \cdots j_{s}^{*}}(t)\right)\right\} \\
& d_{s}(t)=E\left\{\left(q_{\max }-\frac{\varepsilon}{2}-p_{j_{1}^{*} j_{2}^{*} \cdots j_{s}^{*}}(t)\right) \Delta p_{j_{1}^{*} j_{2}^{*} \cdots j_{s}^{*}}(t)\right. \\
& \left.\mid p_{j_{1}^{*} j_{2}^{*} \cdots j_{s}^{*}}(t) \in I_{B}\right\} \\
& e_{s}(t)=E\left\{\Delta^{2} p_{j_{1}^{*} j_{2}^{*} \cdots j_{s}^{*}}(t) \mid p_{j_{1}^{*} j_{2}^{*} \cdots j_{s}^{*}}(t) \in I_{B}\right\} \\
& f_{s}(t)=E\left\{L^{2}\left(p_{j_{1}^{*} j_{2}^{*} \cdots j_{s}^{*}}(t+1)\right) \mid p_{j_{1}^{*} j_{2}^{*} \cdots j_{s}^{*}}(t) \in I_{C}\right\} \text {. }
\end{aligned}
$$

Let us pay the attention to the LA $Z_{j_{1}^{*} j_{2}^{*} \cdots j_{s-1}^{*}}(s=$ $1,2, \ldots, N)$ connected to the optimal path. Concerning the action probability $p_{j_{1}^{*} j_{2}^{*} \cdots j_{s}^{*}}(t)$ related to this automaton, we can prove the following lemma:

Lemma A1: For an arbitrary positive number $\varepsilon$, there exists $\bar{T}(>0)$, and the following inequality (A.2) holds for all $t(>\bar{T})$ :

$$
E\left\{\Delta p_{j_{1}^{*} j_{2}^{*} \cdots j_{s}^{*}}(t) \mid p_{j_{1}^{*} j_{2}^{*} \cdots j_{s}^{*}}(t) \in I_{B}\right\}>0
$$



get

Proof of Lemma A1: By introducing the set $A_{s}(t)$, we can

$$
\begin{aligned}
E\{\Delta & \left.p_{j_{1}^{*} j_{2}^{*} \cdots j_{s}^{*}}(t) \mid p_{j_{1}^{*} j_{2}^{*} \cdots j_{s}^{*}}(t) \in I_{B}\right\} \\
= & E\left\{\Delta p_{j_{1}^{*} j_{2}^{*} \cdots j_{s}^{*}}(t) \mid p_{j_{1}^{*} j_{2}^{*} \cdots j_{s}^{*}}(t) \in I_{B} \wedge j_{s}^{*} \in A_{s}(t)\right\} \\
& \times \operatorname{Pr}\left(j_{s}^{*} \in A_{s}(t)\right) \\
& +E\left\{\Delta p_{j_{1}^{*} j_{2}^{*} \cdots j_{s}^{*}}(t) \mid p_{j_{1}^{*} j_{2}^{*} \cdots j_{s}^{*}}(t) \in I_{B} \wedge j_{s}^{*} \notin A_{s}(t)\right\} \\
& \times \operatorname{Pr}\left(j_{s}^{*} \notin A_{s}(t)\right) \\
= & E\left\{\Delta p_{j_{1}^{*} j_{2}^{*} \cdots j_{s}^{*}}(t) \mid p_{j_{1}^{*} j_{2}^{*} \cdots j_{s}^{*}}(t) \in I_{B} \wedge j_{s}^{*} \in A_{s}(t)\right\} \\
& \times \operatorname{Pr}\left(j_{s}^{*} \in A_{s}(t)\right) .
\end{aligned}
$$

Now let us show that each term of (A.3) is positive. First, we shall consider the first term $E\left\{\Delta p_{j_{1}^{*} j_{2}^{*} \cdots j_{s}^{*}}(t) \mid p_{j_{1}^{*} j_{2}^{*} \ldots j_{s}^{*}}(t)\right.$ $\left.\in I_{B} \wedge j_{s}^{*} \in A_{s}(t)\right\}$. From the fact $v_{j_{1} j_{2} \cdots j_{s}}(t)$ is bounded, and from (9), it can be easily shown that $\left|\Delta p_{j_{1} j_{2} \cdots j_{s}}(t)\right|$ is bounded. This means that there exists $\overline{\Delta p}$ such that $\left|\Delta p_{j_{1} j_{2} \cdots j_{s}}(t)\right|<\overline{\Delta p}$. Furthermore, from (19), $\lambda_{j_{1} j_{2} \cdots j_{s-1}}(t) \rightarrow 0(t \rightarrow \infty)$. Therefore, there exists some $\bar{T}>0$ for an arbitrary positive number $\varepsilon$ and the following inequality (A.4) holds for any $t(>\bar{T})$ :

$$
\begin{aligned}
\lambda_{j_{1} j_{2} \cdots j_{s-1}}(t)\left|\Delta p_{j_{1} j_{2} \cdots j_{s}}(t)\right| & \leq \lambda_{j_{1} j_{2} \cdots j_{s-1}}(t) \overline{\Delta p} \\
& \leq \frac{\varepsilon}{2(r-1)} .
\end{aligned}
$$

Hence, from (8) and (A.4), we can show

$$
\begin{aligned}
& p_{j_{1}^{*} j_{2}^{*} \cdots j_{s-1}^{*} i_{s}}(t+1) \\
& \quad=p_{j_{1}^{*} j_{2}^{*} \cdots j_{s-1}^{*} i_{s}}(t)+\lambda_{j_{1}^{*} j_{2}^{*} \cdots j_{s-1}^{*}}(t) \Delta p_{j_{1}^{*} j_{2}^{*} \cdots j_{s-1}^{*} i_{s}}(t) \\
& \quad \geq p_{j_{1}^{*} j_{2}^{*} \cdots j_{s-1}^{*} i_{s}}(t)-\frac{\varepsilon}{2(r-1)}, \quad t>\bar{T} .
\end{aligned}
$$

Since $p_{j_{1}^{*} j_{2}^{*} \cdots j_{s}^{*}}(t) \leq q_{\max }-(\varepsilon / 2)\left(p_{j_{1}^{*} j_{2}^{*} \cdots j_{s}^{*}}(t) \in I_{B}\right)$, there exists at least one action $\alpha_{j_{1}^{*} j_{2}^{*} \cdots j_{s-1}^{*} i_{s}}\left(i_{s} \neq j_{s}^{*}\right)$ which satisfies

$$
p_{j_{1}^{*} j_{2}^{*} \cdots j_{s-1}^{*} i_{s}}(t) \geq q_{\min }+\frac{\varepsilon}{2(r-1)} .
$$

From this fact and (A.5), we can easily show that there exists at least one action $\alpha_{j_{1}^{*} j_{2}^{*} \cdots j_{s-1}^{*} i_{s}}\left(i_{s} \neq j_{s}^{*}\right)$ for any $t(>\bar{T})$ which satisfies

$$
\begin{aligned}
& p_{j_{1}^{*} j_{2}^{*} \cdots j_{s-1}^{*} i_{s}}(t+1) \geq q_{\min } \\
& \left(p_{j_{1}^{*} j_{2}^{*} \cdots j_{s-1}^{*} i_{s}}(t)-\frac{\varepsilon}{2(r-1)}\right. \\
& \left.\quad \geq q_{\min }+\frac{\varepsilon}{2(r-1)}-\frac{\varepsilon}{2(r-1)}=q_{\min }\right) .
\end{aligned}
$$

This means that there exists at least two components in the set $A_{s}(t)$. Hence, using (9), we can derive the following inequality from the first term of the right-hand side of (A.3):

$$
\begin{aligned}
E & \left\{\Delta p_{j_{1}^{*} j_{2}^{*} \cdots j_{s}^{*}}(t) \mid p_{j_{1}^{*} j_{2}^{*} \cdots j_{s}^{*}}(t) \in I_{B} \wedge j_{s}^{*} \in A_{s}(t)\right\} \\
& =E\left\{v_{j_{1}^{*} j_{2}^{*} \cdots j_{s}^{*}}(t)-\frac{1}{\left|A_{s}(t)\right|} \sum_{l_{s} \in A_{s}(t)} v_{j_{1}^{*} j_{2}^{*} \cdots j_{s-1}^{*} l_{s}}(t)\right\} \\
& =E\left\{v_{j_{1}^{*} j_{2}^{*} \cdots j_{s}^{*}}(t)\right\}-\frac{1}{\left|A_{s}(t)\right|} \sum_{l_{s} \in A_{s}(t)} E\left\{v_{j_{1}^{*} j_{2}^{*} \cdots j_{s-1}^{*} l_{s}}(t)\right\} \\
& >0 .
\end{aligned}
$$

(The last inequality is derived from Lemma 1.)
Now, let us look at the second term $\operatorname{Pr}\left(j_{s}^{*} \in A_{s}(t)\right)$. Since each action of the automaton $Z_{j_{1}^{*} j_{2}^{*} \cdots j_{s-1}^{*}}$ is chosen by the probability larger than $q_{\min }\left(p_{j_{1}^{*} j_{2}^{*} \cdots j_{s-1}^{*} i_{s}}(t) \geq q_{\min }\left(i_{s}=\right.\right.$ $1,2, \ldots, r))$, there is a nonzero probability $\left(\geq\left(q_{\min }\right)^{r}\right)$ that each of the $r$ actions will have been chosen within $r$ steps. Since $E\left\{v_{j_{1}^{*} j_{2}^{*} \cdots j_{s}^{*}}(t)\right\}>E\left\{v_{j_{1}^{*} j_{2}^{*} \cdots j_{s-1}^{*} i_{s}}(t)\right\}\left(i_{s} \neq j_{s}^{*}\right)$ from Lemma 1 , we can get $\operatorname{Pr}\left\{v_{j_{1}^{*} j_{2}^{*} \cdots j_{s}^{*}}(t+r)>v_{j_{1}^{*} j_{2}^{*} \cdots j_{s-1}^{*} i_{s}}(t+r)\right.$ for $\left.{ }^{\forall} i_{s} \neq j_{s}^{*}\right\}>0$ for ${ }^{\forall} t$. Hence, we can obtain

$$
\operatorname{Pr}\left(j_{s}^{*} \in A_{s}(t)\right)>0 .
$$

Therefore, from (A.7) and (A.8), we can prove Lemma A1.

Now, let us examine (A.1). Then, we can get the following relation concerning the action probability $p_{j_{1}^{*} j_{2}^{*} \cdots j_{s}^{*}}(t)$ of the automaton $Z_{j_{1}^{*} j_{2}^{*} \ldots j_{s-1}^{*}}(s=1,2, \ldots, N)$ connected to the optimal path:

$$
\begin{aligned}
b_{s}(t)= & E\left\{L^{2}\left(p_{j_{1}^{*} j_{2}^{*} \cdots j_{s}^{*}}(t)\right)\right\} \\
= & E\left\{L^{2}\left(p_{j_{1}^{*} j_{2}^{*} \cdots j_{s}^{*}}(t)\right) \mid p_{j_{1}^{*} j_{2}^{*} \cdots j_{s}^{*}}(t) \in I_{B}\right\} \\
& \times \operatorname{Pr}\left(p_{j_{1}^{*} j_{2}^{*} \cdots j_{s}^{*}}(t) \in I_{B}\right) \\
& +E\left\{L^{2}\left(p_{j_{1}^{*} j_{2}^{*} \cdots j_{s}^{*}}(t)\right) \mid p_{j_{1}^{*} j_{2}^{*} \cdots j_{s}^{*}}(t) \in I_{C}\right\} \\
& \times \operatorname{Pr}\left(p_{j_{1}^{*} j_{2}^{*} \cdots j_{s}^{*}}(t) \in I_{C}\right) \\
= & E\left\{L^{2}\left(p_{j_{1}^{*} j_{2}^{*} \cdots j_{s}^{*}}(t)\right) \mid p_{j_{1}^{*} j_{2}^{*} \cdots j_{s}^{*}}(t) \in I_{B}\right\} \\
& \times \operatorname{Pr}\left(p_{j_{1}^{*} j_{2}^{*} \cdots j_{s}^{*}}(t) \in I_{B}\right) .
\end{aligned}
$$

By expanding $E\left\{L^{2}\left(p_{j_{1}^{*} j_{2}^{*} \cdots j_{s}^{*}}(t+1)\right)\right\}$, we can get

$$
\begin{aligned}
b_{s}(t+1)= & E\left\{L^{2}\left(p_{j_{1}^{*} j_{2}^{*} \cdots j_{s}^{*}}(t+1)\right)\right\} \\
= & E\left\{L^{2}\left(p_{j_{1}^{*} j_{2}^{*} \cdots j_{s}^{*}}(t+1)\right) \mid p_{j_{1}^{*} j_{2}^{*} \cdots j_{s}^{*}}(t) \in I_{B}\right\} \\
& \times \operatorname{Pr}\left(p_{j_{1}^{*} j_{2}^{*} \cdots j_{s}^{*}}(t) \in I_{B}\right) \\
& +E\left\{L^{2}\left(p_{j_{1}^{*} j_{2}^{*} \cdots j_{s}^{*}}(t+1)\right) \mid p_{j_{1}^{*} j_{2}^{*} \cdots j_{s}^{*}}(t) \in I_{C}\right\} \\
& \times \operatorname{Pr}\left(p_{j_{1}^{*} j_{2}^{*} \cdots j_{s}^{*}}(t) \in I_{C}\right) .
\end{aligned}
$$

By utilizing (8), (9), and (A.1), we can derive the following equality concerning the first term of the right-hand side:

$$
\begin{aligned}
E\{ & \left.L^{2}\left(p_{j_{1}^{*} j_{2}^{*} \cdots j_{s}^{*}}(t+1)\right) \mid p_{j_{1}^{*} j_{2}^{*} \cdots j_{s}^{*}}(t) \in I_{B}\right\} \\
= & E\left\{\left(q_{\max }-\frac{\varepsilon}{2}-p_{j_{1}^{*} j_{2}^{*} \cdots j_{s}^{*}}(t)\right.\right. \\
& \left.\left.-\lambda_{j_{1}^{*} j_{2}^{*} \cdots j_{s-1}^{*}}(t) \Delta p_{j_{1}^{*} j_{2}^{*} \cdots j_{s}^{*}}(t)\right)^{2} \mid p_{j_{1}^{*} j_{2}^{*} \cdots j_{s}^{*}}(t) \in I_{B}\right\} \\
= & E\left\{\left(q_{\max }-\frac{\varepsilon}{2}-p_{j_{1}^{*} j_{2}^{*} \cdots j_{s}^{*}}(t)\right)^{2} \mid p_{j_{1}^{*} j_{2}^{*} \cdots j_{s}^{*}}(t) \in I_{B}\right\} \\
& -2 \lambda_{j_{1}^{*} j_{2}^{*} \cdots j_{s-1}^{*}}(t) E\left\{\left(q_{\max }-\frac{\varepsilon}{2}-p_{j_{1}^{*} j_{2}^{*} \cdots j_{s}^{*}}(t)\right)\right. \\
& \left.\times \Delta p_{j_{1}^{*} j_{2}^{*} \cdots j_{s}^{*}}(t) \mid p_{j_{1}^{*} j_{2}^{*} \cdots j_{s}^{*}}(t) \in I_{B}\right\} \\
& +\lambda_{j_{1}^{*} j_{2}^{*} \cdots j_{s-1}^{*}}^{2}(t) E\left\{\Delta^{2} p_{j_{1}^{*} j_{2}^{*} \cdots j_{s}^{*}}(t) \mid p_{j_{1}^{*} j_{2}^{*} \cdots j_{s}^{*}}(t) \in I_{B}\right\} \\
= & E\left\{L^{2}\left(p_{j_{1}^{*} j_{2}^{*} \cdots j_{s}^{*}}(t)\right) \mid p_{j_{1}^{*} j_{2}^{*} \cdots j_{s}^{*}}(t) \in I_{B}\right\} \\
& \left.-2 \lambda_{j_{1}^{*} j_{2}^{*} \cdots j_{s-1}^{*}}(t) d_{s}(t)+\lambda_{j_{1}^{*} j_{2}^{*} \cdots j_{s-1}^{*}}^{2}(t) e_{s}(t) . \quad \text { (A. } 11\right)
\end{aligned}
$$


Hence, from (A.10) and (A.11), we can get

$$
\begin{array}{r}
b_{s}(t+1)=f_{s}(t) P_{s C}(t)+b_{s}(t)+P_{s B}(t)\left(\lambda_{j_{1}^{*} j_{2}^{*} \cdots j_{s-1}^{*}}^{2}(t) e_{s}(t)\right. \\
\left.-2 \lambda_{j_{1}^{*} j_{2}^{*} \cdots j_{s-1}^{*}}(t) d_{s}(t)\right) . \quad(\mathrm{A} .12)
\end{array}
$$

From the above equation, we can derive

$$
\begin{aligned}
& 2 \sum_{k=1}^{t} P_{s B}(k) \lambda_{j_{1}^{*} j_{2}^{*} \cdots j_{s-1}^{*}}(k) d_{s}(k)=b_{s}(1)-b_{s}(t+1)+\sum_{k=1}^{t} \\
& \quad \times f_{s}(k) P_{s C}(k)+\sum_{k=1}^{t} P_{s B}(k) \lambda_{j_{1}^{*} j_{2}^{*} \cdots j_{s-1}^{*}}^{2}(k) e_{s}(k) . \quad \text { (A.13) }
\end{aligned}
$$

Since $b_{s}(t) \geq 0$ for an arbitrary time $t$, we can get from (A.13)

$$
\begin{array}{r}
2 \sum_{k=1}^{\infty} P_{s B}(k) \lambda_{j_{1}^{*} j_{2}^{*} \cdots j_{s-1}^{*}}(k) d_{s}(k) \leq b_{s}(1)+\sum_{k=1}^{\infty} f_{s}(k) P_{s C}(k) \\
+\sum_{k=1}^{\infty} P_{s B}(k) \lambda_{j_{1}^{*} j_{2}^{*} \cdots j_{s-1}^{*}}^{2}(k) e_{s}(k) . \quad \text { (A.14) }
\end{array}
$$

Furthermore, $\lambda_{j_{1}^{*} j_{2}^{*} \cdots j_{s-1}^{*}}^{2}(k) e_{s}(k) \leq \lambda_{j_{1}^{*} j_{2}^{*} \cdots j_{s-1}^{*}}^{2}(k)(\overline{\Delta p})^{2}$ and $f_{s}(k) \leq \lambda_{j_{1}^{*} j_{2}^{*} \cdots j_{s-1}^{*}}^{2}(k)(\Delta \bar{p})^{2}$. Therefore, it can be easily shown from (19) that the two infinite series of the right-hand side of (A.14) converge. Hence, $\sum_{k=1}^{\infty} P_{s B}(k) \lambda_{j_{1}^{*} j_{2}^{*} \cdots j_{s-1}^{*}}(k) d_{s}(k)$ is bounded above.

Now that we have already proven Lemma A1, let us choose one $\bar{T}$ which satisfies (A.2). Then, from the fact that $\lambda_{j_{1}^{*} j_{2}^{*} \cdots j_{s-1}^{*}}(t) d_{s}(t) \geq 0$ for an arbitrary time $t(t>\bar{T})$, it can be easily shown that $\sum_{k=\bar{T}}^{\infty} P_{s B}(k) \lambda_{j_{1}^{*} j_{2}^{*} \cdots j_{s-1}^{*}}(k) d_{s}(k)$ is bounded above.

Hence, we can arrive at the following:

$\sum_{k=1}^{\infty} P_{s B}(k) \lambda_{j_{1}^{*} j_{2}^{*} \cdots j_{s-1}^{*}}(k) d_{s}(k)$ exists and has a finite value.

By following the same procedure as in [20, p. 397], we can obtain (20).

Q.E.D.

\section{ACKNOWLEDGMENT}

The authors would like to thank the Reviewers for their suggestions and comments.

\section{REFERENCES}

[1] M. L. Tsetlin, "On the behavior of finite automata in random media," Avtomatika i Telemekhanika, vol. 22, no. 10, pp. 1345-1354, 1961.

[2] V. I. Varshavskii and I. P. Vorontsova, "On the behavior of stochastic automata with variable structure," Autom. Remote Control, vol. 24, pp. 327-333, 1963.

[3] S. Lakshmivarahan, Learning Algorithms Theory and Applications. New York: Springer-Verlag, 1981.

[4] K. S. Narendra and M. A. L. Thathachar, "Learning automata-A survey," IEEE Trans. Syst., Man, Cybern., vol. SMC-4, pp. 323-334, July 1974

[5] N. Baba, New Topics in Learning Automata Theory and Applications. New York: Springer-Verlag, 1985.

[6] K. S. Narendra and M. A. L. Thathachar, Learning Automata: An Introduction. Englewood Cliffs, NJ: Prentice-Hall, 1989.

[7] A. S. Poznyak and K. Najim, Learning Automata and Stochastic Optimization. New York: Springer-Verlag, 1997.

[8] M. A. L. Thathachar and K. R. Ramakrishnan, "A hierarchical system of learning automata," IEEE Trans. Syst., Man, Cybern., vol. SMC-11, pp. 236-241, Mar. 1981.

[9] K. R. Ramakrishnan, "Hierarchical systems and cooperative games of learning automata," Ph.D. dissertation, Indian Institute of Science, Bangalore, India, 1982.
[10] N. Baba, "Learning behaviors of hierarchical structure stochastic automata operating in a general multiteacher environment," IEEE Trans. Syst., Man, Cybern., vol. SMC-15, pp. 585-587, July/Aug. 1985.

[11] M. A. L. Thathachar and V. V. Phansalkar, "Learning the global maximum with parameterized learning automata," IEEE Trans. Neural Networks, vol. 6, pp. 398-406, Mar. 1995.

[12] _ - "Convergence of teams and hierarchies of learning automata in connectionist systems," IEEE Trans. Syst., Man, Cybern., vol. 25, pp. 1459-1469, Nov. 1995.

[13] M. A. L. Thathachar and P. S. Sastry, "A class of rapidly converging algorithms for learning automata," in Proc. IEEE Int. Conf. Cybernetics and Society, Bombay, India, 1984, pp. 602-606.

[14] - "A new approach to the design of reinforcement schemes of learning automata," IEEE Trans. Syst., Man, Cybern., vol. SMC-15, pp. 168-175, Jan./Feb. 1985.

[15] —, "A hierarchical system of learning automata that can learn the globally optimal path," Inf. Sci., vol. 42, pp. 143-166, 1987.

[16] B. J. Oommen and J. K. Lanctot, "Discretized pursuit learning automata," IEEE Trans. Syst., Man, Cybern., vol. 20, pp. 931-938, July/Aug. 1990.

[17] G. I. Papadimitriou, "A new approach to the design of reinforcement schemes for learning automata: Stochastic estimator learning algorithms," IEEE Trans. Knowledge Data Eng., vol. 6, pp. 649-654, Aug. 1994.

[18] - "Hierarchical discretized pursuit nonlinear learning automata with rapid convergence and high accuracy," IEEE Trans. Knowledge Data Eng., vol. 6, pp. 654-659, Aug. 1994.

[19] B. J. Oommen and M. Agache, "Continuous and discretized pursuit learning scheme: Various algorithms and their comparison," IEEE Trans. Syst., Man, Cybern. B, vol. 31, pp. 277-287, June 2001.

[20] R. Simha and J. F. Kurose, "Relative reward strength algorithms for learning automata," IEEE Trans. Syst., Man, Cybern., vol. 19, pp. 388-398, Mar./Apr. 1989

[21] M. A. L. Thathachar and M. T. Arvind, "Parallel algorithms for modules of learning automata," IEEE Trans. Syst., Man, Cybern. B, vol. 28, pp. 24-33, Feb. 1998

[22] P. R. Srikantakumar and K. S. Narendra, "A learning model for routing in telephone networks," SIAM J. Control Optim., vol. 20, pp. 34-57, 1982.

[23] N. Baba and Y. Sawaragi, "On the learning behavior of stochastic automata under a nonstationary random environment," IEEE Trans. Syst., Man, Cybern., vol. SMC-5, pp. 273-275, Mar. 1975.

[24] N. Baba, "On the learning behaviors of variable-structure stochastic automaton in the general N-teacher environment," IEEE Trans. Syst., Man, Cybern., vol. SMC-13, pp. 224-231, Mar./Apr. 1983.

[25] N. Baba and Y. Mogami, "Learning behaviors of hierarchical structure stochastic automata in a nonstationary multiteacher environment," Int. J. Syst. Sci., vol. 19, pp. 1345-1350, 1988.

[26] X. Zeng, J. Zhou, and C. Vasseur, "A strategy for controlling nonlinear systems using a learning automaton," Automatica, vol. 36, pp. $1517-1524,2000$.

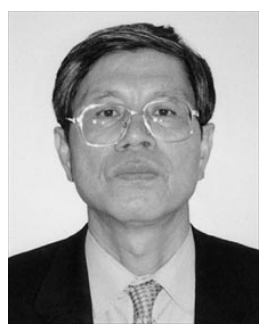

Norio Baba (S'74-M'74) received the B.E., M.E., and Dr.Eng. degrees in applied mathematics and physics from Kyoto University, Kyoto, Japan, in 1969,1971 , and 1975, respectively.

$\mathrm{He}$ joined the University of Tokushima, Tokushima, Japan, as an Assistant Professor in 1974, and became a Professor of Intelligent Information Engineering Systems at the same university in June 1988. He was invited by INRIA, France and IIASA, Austria as a Visiting Research Fellow in 1979 and 1983, respectively. Since April 1990, he has been a Professor of Information Science at Osaka Kyoiku University, Kashiwara City, Japan. His research interests include learning automata, neural networks, genetic algorithms, computer gaming, financial engineering, robotics, stochastic programming, and computer pets. He has authored or coauthored more than 150 technical journal and conference papers. He is the author of New Topics in Learning Automata Theory and Applications (New York: Springer-Verlag, 1985). He is also the coeditor of the books Computational Intelligence in Games (Heidelberg, Germany: Physica-Verlag, 2001), and Knowledge-Based Intelligent Information Engineering Systems and Allied Technologies (Amsterdam, The Netherlands: IOS Press, 2001).

Dr. Baba was the Chairman of the Fifth International Conference on Knowledge-Based Intelligent Information Engineering Systems and Allied Technologies (September, 2001). He is currently on the Editorial Advisory Board of International Journal of Knowledge-Based Intelligent Engineering Systems. 


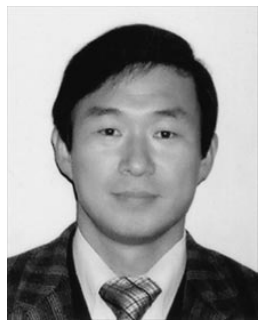

Yoshio Mogami (M'96) received the B.E. and M.E. degrees in mechanical engineering from Kyoto Institute of Technology, Kyoto, Japan, in 1973 and 1975, respectively, and the Dr.Eng. degree in applied mathematics and physics from Kyoto University in 1996.

From 1975 to 1997, he was with the University of Tokushima, Tokushima, Japan, as an Assistant Professor in the Faculty of Engineering, and, since 1997, he has been an Associate Professor in the same faculty. His current research interests include HSLA, variable hierarchical structure learning automata, reinforcement learning, and optimization problems. 